\title{
Commissioning of the CUORE Cryostat: The First Experimental Setup for Bolometric Detectors at the 1 Ton Scale
}

\author{
Nicholas I. Chott* \\ Department of Physics and Astronomy, University of South Carolina, Columbia, SC 29208 - USA \\ E-mail: chotteemail.sc.edu; nchottegmail.com
}

\section{Franco Alessandria}

INFN - Sezione di Milano, Milano I-20133 - Italy

\section{Carlo Bucci; Paolo Gorla}

INFN - Laboratori Nazionali del Gran Sasso, Assergi (L’Aquila) I-67010 - Italy

\section{Luigi Cappelli; Carmine Pagliarone}

Dipartimento di Ingegneria Civile e Meccanica, Università degli Studi di Cassino e del Lazio Meridonale, Cassino I-03043 - Italy

INFN - Laboratori Nazionali del Gran Sasso, Assergi (L'Aquila) I-67010 - Italy

\section{Carlo Ligi}

INFN - Laboratori Nazionali di Frascati, Frascati (Roma) I-00044 - Italy

\section{Angelo Nucciotti; Francesco Terranova}

Dipartimento di Fisica, Università di Milano-Bicocca, Milano I-20126 - Italy

INFN - Sezione di Milano Bicocca, Milano I-20126 - Italy

\section{Luca Taffarello}

INFN - Sezione di Padova, Padova I-35131 - Italy

The Cryogenic Underground Observatory for Rare Events (CUORE) is a 1-ton scale bolometric experiment. The CUORE detector is an array of $988 \mathrm{TeO}_{2}$ crystals arranged in a cylindrical compact structure of 19 towers. This will be by far the largest bolometric mass ever operated. These detectors need a base temperature around $10 \mathrm{mK}$ in order to meet the performance specifications. To cool the CUORE detector a large cryogenic-free cryostat with five pulse tubes and one custom high-power dilution refrigerator has been designed. The cryostat ( $4 \mathrm{~K}$ refrigerator with pulse tubes) and dilution unit were first tested independently and then merged together. We report here a detailed description of the cryostat for the CUORE experiment together with the results of the validation tests done in 2014.

Technology and Instrumentation in Particle Physics 2014,

2-6 June, 2014

Amsterdam, the Netherlands

\footnotetext{
* Speaker.
} 


\section{Searching for Neutrinoless Double Beta $(0 \vee \beta \beta)$ Decay with CUORE}

Experimental observation of neutrino oscillations confirm they have mass but important properties of the neutrino remain unknown. Like missing puzzle pieces, a clear picture of neutrinos and their properties have yet to be realized such as the Dirac/Majorana nature of the neutrino, the mass hierarchy and the absolute neutrino masses. Neutrinoless double beta decay experiments, like CUORE, play a crucial role in advancing our understanding of neutrino properties. Only these experiments are able to probe the Majorana nature of the neutrino $(\bar{v}=v)$. These experiments can also give information about the neutrino mass hierarchy and absolute mass scale. The primary goal of the Cryogenic Underground Observatory for Rare Events (CUORE) experiment at the Laboratori Nazionali del Gran Sasso (LNGS), is the search for neutrinoless double beta decay but it also aims to be sensitive enough to search for dark matter and solar axions.

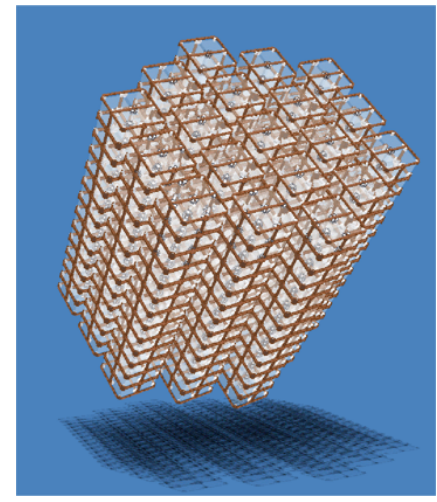

Figure 1: Artist rendering of the CUORE detector.

CUORE is a low temperature bolometric detector in which the source and detector are one and the same. This "source $=$ detector" technique ensures a high detector efficiency. The $0 v \beta \beta$ decay candidate chosen for CUORE is ${ }^{130} \mathrm{Te}$. Tellurium is selected for both element specific and isotope specific reasons. Not only does it have a large natural abundance of the double beta decay isotope ${ }^{130} \mathrm{Te}$ ( $\sim 34 \%$ ) with high Q-value ( $2528 \mathrm{keV})$, it also has a favorable nuclear factor of merit. The geometry of the CUORE detector array is a cylindrical structure of 19 closely packed towers, as seen in figure 1 . There are 13 floors for each tower, consisting of $45 \times 5 \times 5 \mathrm{~cm}^{3} \mathrm{TeO}_{2}$ crystals $(750 \mathrm{~g}$ each). There are 988 crystals with a total mass of $741 \mathrm{~kg}$ of $\mathrm{TeO}_{2}\left(204 \mathrm{~kg}\right.$ of $\left.{ }^{130} \mathrm{Te}\right)$. To demonstrate the feasibility of this ambitious project a single CUORE tower, named CUORE-0, has been constructed and is currently running in Hall A of LNGS.

\section{CUORE Cryostat Design Requirements}

The experimental sensitivity to detect $0 v \beta \beta$ decay is shown in equation 2.1,

$$
S^{0 v} \propto \frac{\varepsilon a_{i}}{A} \sqrt{\frac{M t}{b \Delta E}}, \quad b \neq 0
$$

were $\varepsilon$ is the detector efficiency, $\mathrm{a}_{i}$ is the isotopic abundance, $\mathrm{A}$ is the mass number, $\mathrm{M}$ is the total active mass (in $\mathrm{kg}$ ), $\mathrm{t}$ is the time in years, $\mathrm{b}$ is the background (count $/ \mathrm{kg} / \mathrm{keV} / \mathrm{y}$ ) and $\Delta \mathrm{E}$ is the energy resolution (keV) in the $0 v \beta \beta$ decay region of interest (ROI). The parameters under the square root are crucial to the design of the CUORE cryostat to maximize experimental sensitivity.

\subsection{Active Mass}

One way to increase experimental sensitivity is to build a larger detector to increase the number of ${ }^{130} \mathrm{Te}$ nuclei. The CUORE detector is $741 \mathrm{~kg}$ of $\mathrm{TeO}_{2}\left(204 \mathrm{~kg}{ }^{130} \mathrm{Te}\right)$. The dimensions of the experimental space required is $0.9 \mathrm{~m}$ in diameter and $1.385 \mathrm{~m}$ high. In order to contain such a large detector a dedicated cryostat was constructed, with the outermost (300 K) vessel $3.1 \mathrm{~m}$ high and 
$1.687 \mathrm{~m}$ in diameter. The cryostat needs to be powerful enough to cool down a large mass to a stable temperature of $10 \mathrm{mK}$ and account for the thermal radiation of the shields.

\subsection{Background reduction}

The background, especially in the $0 v \beta \beta$ decay ROI, should be as low as possible. The CUORE target background is $<10^{-2}$ counts $/ \mathrm{keV} / \mathrm{kg} /$ year. Hall A of LNGS has an average depth of 3650 meter water equivalent (m.w.e) with a $\mu$ flux of $(2.85 \pm 0.3) \times 10^{-8} \mu / \mathrm{s} / \mathrm{cm}^{2}$, a neutron flux $(<10$ $\mathrm{MeV})$ of $4 \times 10^{-6} \mathrm{n} / \mathrm{s} / \mathrm{cm}^{2}$ and a $\gamma$ flux $(<3 \mathrm{meV})$ : of $0.73 \gamma / \mathrm{s} / \mathrm{cm}^{2}$. In addition to operating CUORE underground, a strict selection of low radioactive materials are used for cryostat construction. To protect the detector from radioactive contamination of materials within the cryostat that cannot be excluded $\sim 10$ tons of lead shielding ( $\mathrm{Pb}+\mathrm{Cu}$ OFE supports) will be contained inside the cryogenic space and maintained at temperatures of $4 \mathrm{~K}$ or less.

\subsection{Energy Resolution}

Energy resolution of the detector is crucial in order to see the $0 v$ peak over the $2 v$ continuum. Bolometers have very good energy resolution of $\sim 5 \mathrm{keV}$ FWHM at the ${ }^{208} \mathrm{Tl}$ peak $(2615 \mathrm{keV}$ ) [1]. This peak is less than $100 \mathrm{keV}$ away from the $0 v \beta \beta$ decay $\mathrm{Q}$ value of ${ }^{130} \mathrm{Te}(\sim 2527 \mathrm{keV})$. In order to maintain this energy resolution in CUORE the cryostat will need to have a stable operating temperature of $10 \mathrm{mK}$ to ensure optimal performance of the $\mathrm{TeO}_{2}$ crystals and the NTDs. In addition, NTDs have a very high impedance so the level of vibrations transmitted to the detector needs to be on the order of the energy resolution, roughly $1 \mathrm{keV}\left(\sim 10^{-16} \mathrm{~J}\right)$, to avoid micro-phonic noise. Sources of vibrational noise include pumps, compressors, pulse tubes and the dilution unit.

\subsection{Time}

CUORE will run for at least 5 years and during this time the cryostat needs to be stable and relatively service-free to maximize live time. The CUORE- 0 cryostat has a main bath that needs to be refilled every two days with liquid helium, this reduces live time by $\sim 7 \%$ (roughly 3 hours every 2 days). The CUORE- 0 cryostat also contains a $1 \mathrm{~K}$ pot which introduces noise in the baseline of the detectors from the evaporation of helium. In light of this past experience the CUORE cryostat is designed to be cryogen free, to improve live time and reduce noise.

\section{CUORE Cryostat Description}

The CUORE cryostat layout is shown in Figure 2. There are a total of 6 thermal shields at temperatures of $300 \mathrm{~K}, 40 \mathrm{~K}$, $4 \mathrm{~K}, 800 \mathrm{mK}, 50 \mathrm{mK}$ and $10 \mathrm{mK}$. Two of these shields are vacuum chambers $(300 \mathrm{~K}$ and $4 \mathrm{~K}$ ) to allow for the use of exchange gas in the inner vacuum chamber (IVC) during the initial stage of cooling. 5 pulse tube refrigerators are mounted to the $300 \mathrm{~K}$ flange to

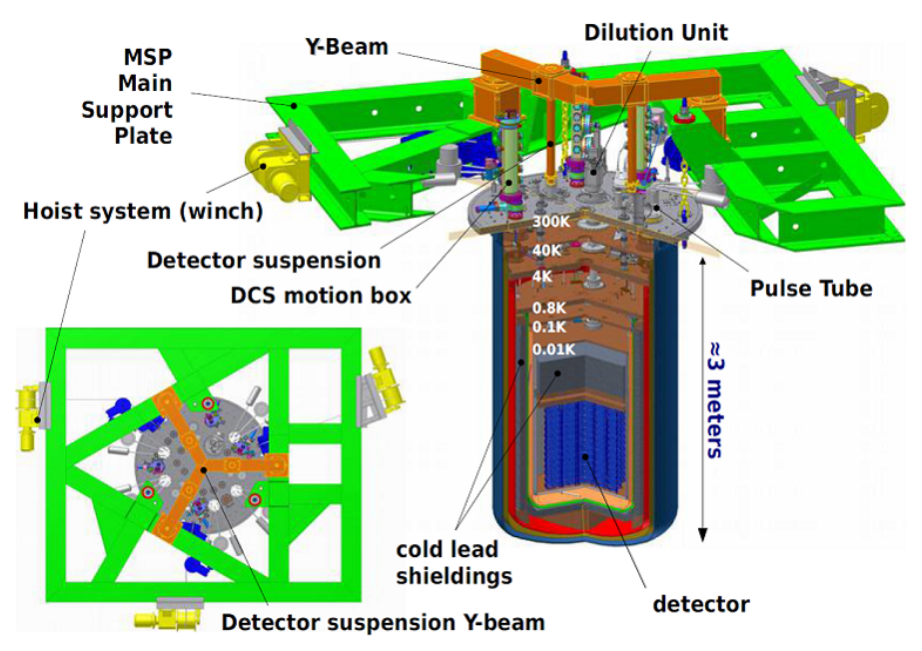

3 Figure 2: Schematic of the CUORE cryostat. 
ensure a higher live time, eliminating the need to refill the main bath. The 5 outer cryostat flanges and vessels are made of $\mathrm{Cu}-\mathrm{OFE}$, with the exception if the $300 \mathrm{~K}$ flange and upper part of the $300 \mathrm{~K}$ vessel, made of 304L stainless steel which is better for mechanical stability and vacuum tightness. The mixing chamber flange and vessel, as well as the detector structure, is made of Cu NOSV. A Helicoflex model HNV 200 is used to create the 4K vacuum seal [2]. Cryostat flanges are fixed in place with a dedicated hoist system to raise and lower the shields. The entire cryostat is suspended from a main support plate (MSP) which will bear the load of close to 20 tons, specifically the detector $(\sim 1$ ton), thermal shields and flanges ( $\sim 8$ tons) and the internal lead shielding ( $\sim 10$ tons). To minimize vibrational noise of the detector as much as possible there are independent suspensions for the vessels, dilution unit, and detector. Support bars of are made of $316 \mathrm{LN}$ steel. The detector suspension is a two stage low-frequency isolator fixed to a y-beam above the cryostat. The first stage is a negative stiffness mechanism made by Minus- $\mathrm{K}$ and the second stage consists regular springs at the top of the suspension bars.
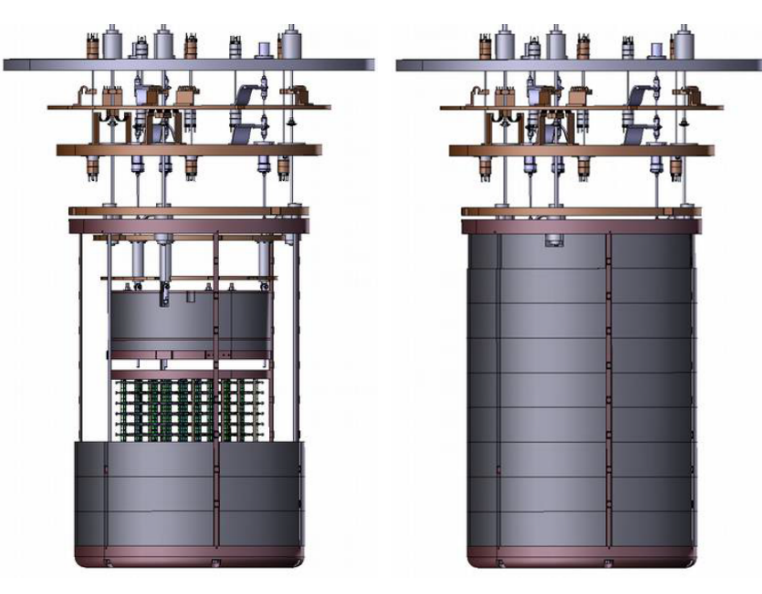

Figure 3: Schematic of the top and lateral cold lead shielding of the CUORE detector.
The cold lead shielding, seen in figure 3 , is made of two parts: the top lead and lateral lead shield. It is designed, together with the external lead shield, to surround the detector with $30 \mathrm{~cm}$ of lead in all directions. The top lead is a disc of modern lead $(2745 \mathrm{~kg}) 30 \mathrm{~cm}$ thick located below the mixing chamber (MC) plate and thermalized to the $50 \mathrm{mK}$ stage. The top lead will shield the detector from the upper cryostat while a $10 \mathrm{~cm}$ thick ultra-pure copper disc is mounted below the top lead to shield the detectors from ${ }^{210} \mathrm{~Pb}$, present in the top lead. The lateral lead consists of several $6 \mathrm{~cm}$ thick annular sections of ancient Roman lead $(5562 \mathrm{~kg}$ ) located between the $4 \mathrm{~K}$ and Still shields and thermalized at a temperature of $4 \mathrm{~K}$. There are dedicated supports from $300 \mathrm{~K}$ flange to bear the load of the top lead, while the Still suspension will support the load of the lateral lead. Ancient Roman lead, salvaged from a sunken ship, is ideal for low background experiments due to the low activity of ${ }^{210} \mathrm{~Pb}(<4 \mathrm{mBq} / \mathrm{kg})$ [3].

\section{Cooling Methods of the CUORE Cryostat}

\subsection{Pulse Tube Refrigerators}

The CUORE cryostat is equipped with 5 pulse tube (PT) refrigerators as seen in figure 4 . Pulse tubes are used in the initial cooling of the cryostat and to maintain the temperature of the $40 \mathrm{~K}$ and $4 \mathrm{~K}$ stages. Each pulse tube is a commercially available two stage Cryomech model PT415 with a cooling power of $40 \mathrm{~W}$ at the $40 \mathrm{~K}$ stage and $1.5 \mathrm{~W}$ at $4 \mathrm{~K}$. The remote motor option is utilized to reduce vibrational noise transmitted
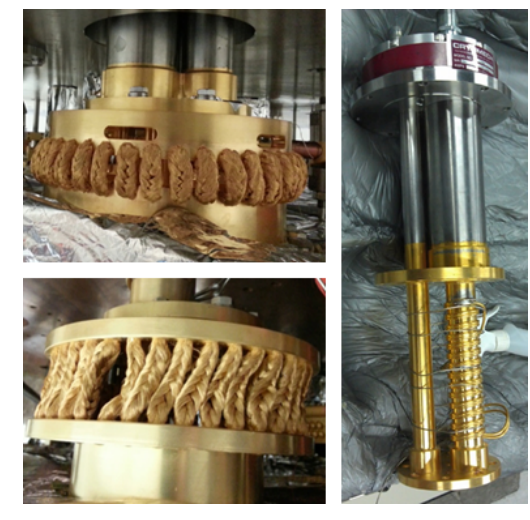

Figure 4: CUORE pulse tube and copper thermalization at $40 \mathrm{~K}$ and $4 \mathrm{~K}$ stage. 
to the detector and preserve energy resolution. Since cooling power of the PTs are diminished as the motor is moved farther away, it is mounted $60 \mathrm{~cm}$ away to optimize the cooling power while still reducing vibrational noise. Flexible thermalizations (gilded copper braids) connect both cold heads of each PT to the cryostat flanges at the $40 \mathrm{~K}$ and $4 \mathrm{~K}$ stage. Finally around the head of each $\mathrm{PT}$, mounted directly to the $300 \mathrm{~K}$ plate, is a rubber ring to further dampen vibrations.

\subsection{Dilution Unit}

The CUORE dilution unit (DU) is a custom designed (modified DRS-CF2000) high-power Joule-Thompson DU from Leiden Cryogenics. It is designed for high circulation rates of ${ }^{3} \mathrm{He} /{ }^{4} \mathrm{He}$ mixture and contains $120 \mathrm{~L}$ of ${ }^{3} \mathrm{He}$. The condensing lines of the incoming mixture are thermalized around the cold stages of 2PTs. There is no need for a $1 \mathrm{~K}$ pot because in normal operation the Still behaves like one to cool the incoming mixture. The DU is designed for two distinct modes, the first for high cooling power and the second for operating at a low base temperature. The condensing stage (two for redundancy) is equipped with a variable impedance system to regulate the mixture flow and switch between the two operational modes. Initially the inner stages are all at a temperature of $4 \mathrm{~K}$ and a high mixture flow of $8 \mathrm{mmol} / \mathrm{s}$ quickly cools several tons of mass down to the respective operating temperature of each stage. At this point the mixture flow is reduced to less than $1 \mathrm{mmol} / \mathrm{s}$ by adjusting the impedance. A low mixture flow is required to reach $10 \mathrm{mK}$ in order to operate the detector because at base temperature most of the heat on the mixing chamber (MC) stage comes from the incoming mixture.

\subsection{Fast Cooling System}

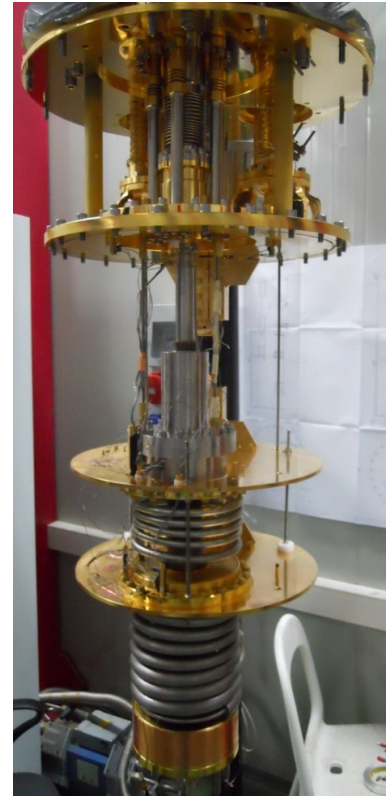

Figure 5: CUORE dilution unit mounted in test cryostat.
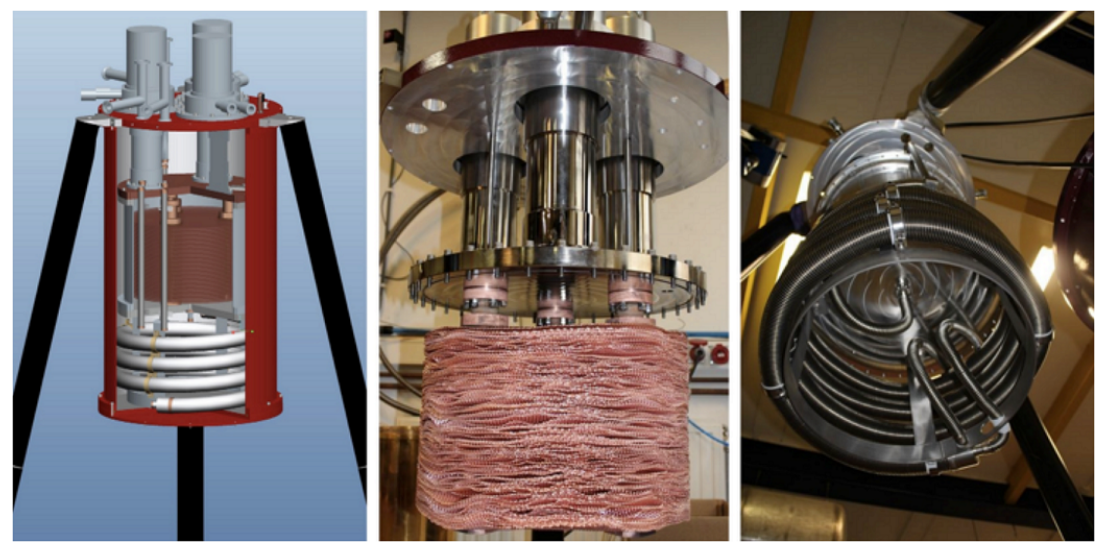

Figure 6: Fast Cooling System [left] and both the copper mesh [center] and counter flow [right] heat exchangers.

Pulse tubes alone would take close to 5 months to cool the full IVC mass to a temperature of $4 \mathrm{~K}$ so a fast cooling system (FCS) was developed to quickly cool $\sim 15$ tons of material in the IVC 
down to $\sim 40 \mathrm{~K}$. The FCS, figure 6, is an external vessel with two heat exchangers and three GiffordMcMahon cryo-coolers, each with a cooling power of $600 \mathrm{~W}$ at $77 \mathrm{~K}$. Cooling is achieved through a forced circulation of cold helium gas, progressively cooled in the external vessel and injected directly in to the cryostat IVC. To avoid thermal shock to the detector (and the cryostat), $\Delta \mathrm{T}$ of the incoming and outgoing gas should not exceed $40 \mathrm{~K}$. Estimated cooldown time to $4 \mathrm{~K}$ (FCS + PTs) is about a week and assumes the full IVC mass: 6 tons of copper (shields and lead supports) and 8.3 tons of lead, a realistic thermal coupling of the 5 PTs, and a perfect thermal coupling for the 3 GMs and the FCS heat exchangers. Expected cooldown time for normal operation will be between $1-2$ weeks so as to not stress the system. Commissioning of the FCS at Leiden Cryogenics is in the final stage and delivery to LNGS is expected July 2014.

\section{CUORE Cryostat Commissioning}

The commissioning of the CUORE cryostat is divided in to two main phases. The first phase involves independent commissioning of both the cryostat (to $4 \mathrm{~K}$ ) and the dilution unit in parallel. In the second phase, the DU and cryostat are merged for the commissioning of the complete cryogenic system.

\subsection{K Cryostat}

The 4 K CUORE cryostat was commissioned with 3/5 PTs installed and the $300 \mathrm{~K}$, $40 \mathrm{~K}$ and $4 \mathrm{~K}$ flanges and vessels. Two of these vessels are vacuum chambers, the outer vacuum chamber (OVC) at $300 \mathrm{~K}$ and the inner vacuum chamber (IVC) at $4 \mathrm{~K}$. Two $4 \mathrm{~K}$ cold tests were performed during this phase. The first run was with "no load" (only $4 \mathrm{~K}$ flange/vessel $\sim 2$ tons of $\mathrm{Cu}$ ) and the second run, dedicated to detector calibration system (DCS) cold tests, had a temporary stainless steel beam mounted below the $4 \mathrm{~K}$ plate. The results of these cold tests are very promising, a stable temperature of $32 \mathrm{~K}$ was reached at the $40 \mathrm{~K}$ stage and $3.3 \mathrm{~K}$ at the $4 \mathrm{~K}$ stage.

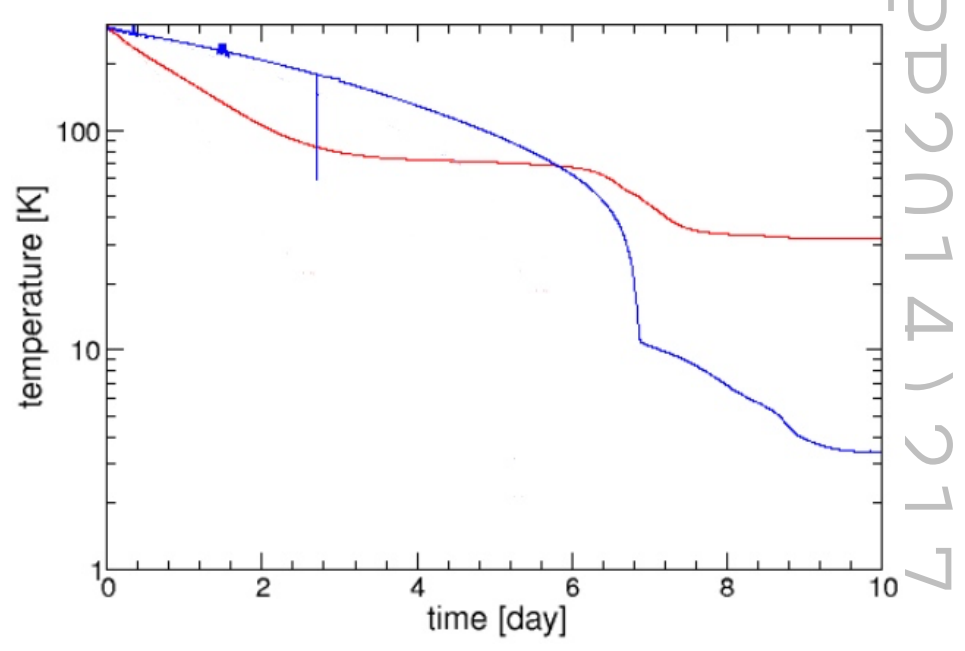

Figure 7: Temperature of the $40 \mathrm{~K}$ stage and $4 \mathrm{~K}$ as a function of time. Stable temperature of $32 \mathrm{~K}$ reached at the $40 \mathrm{~K}$ stage and $3.3 \mathrm{~K}$ at the $4 \mathrm{~K}$ stage with 3 pulse tubes.

Roughly 9 days were required to reach a stable temperature at each stage using 3PTs. The commissioning of the CUORE $4 \mathrm{~K}$ cryostat was completed in October 2013 after meeting the required design specifications. The tail at the end of the $4 \mathrm{~K}$ stage temperature in figure 7 is due to the thermalization of the stainless steel beam and will not be present in CUORE.

\subsection{Dilution Unit}

The CUORE dilution unit was first tested at Leiden Cryogenics in a dedicated test cryostat where a base temperature of $5.26 \mathrm{mK}$ was achieved with a MC cooling power of $2 \mathrm{~mW}$ at $100 \mathrm{mK}$ ( $3 \mathrm{~mW}$ at $123 \mathrm{mK}$ ). At LNGS, the test cryostat was setup in the ground floor of the CUORE hut 
and a base temperature of $4.95 \mathrm{mK}$ was achieved. Characterization tests of the DU were preformed by injecting power on the cold stages, as seen in figure 8. Power on the Still and the heat exchange (HEX) stages were fixed and MC temperature as a function of cooling power measured.

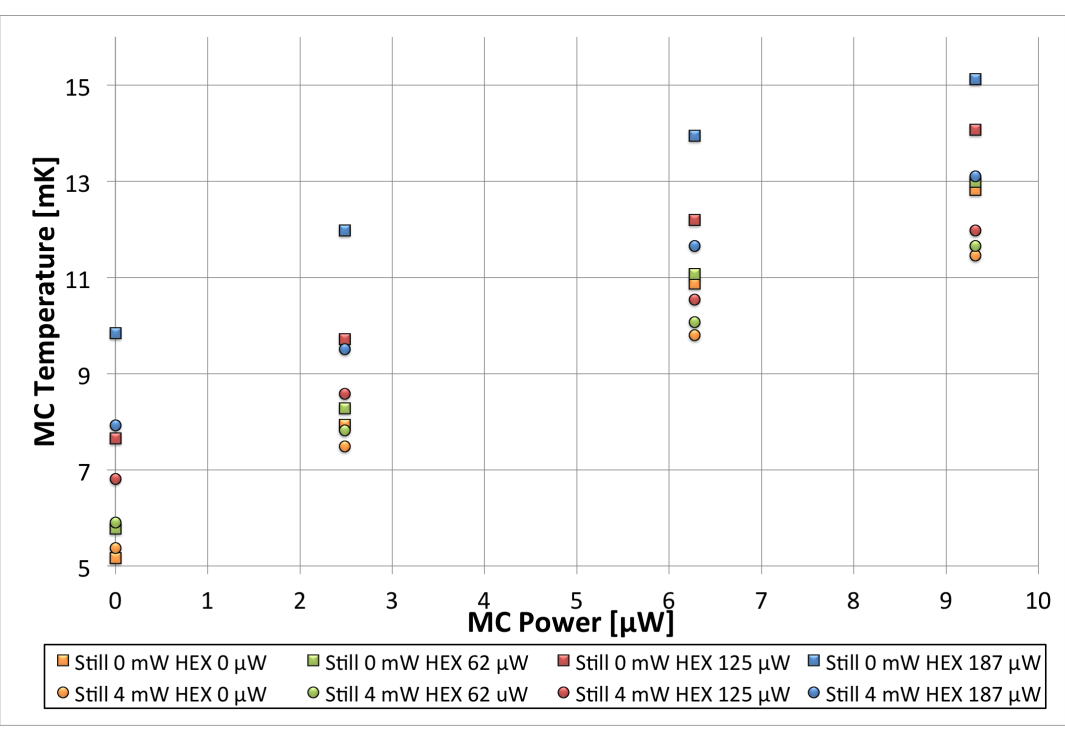

Figure 8: Characterization of the dilution unit. Different combinations of power injected on Still and Cold Plate/HEX and temperature of the mixing chamber as a function of cooling power measured.
The power specifications of the DU was for a MC cooling power of $5 \mu \mathrm{W}$ at $12 \mathrm{mK}$, with $3 \mathrm{~mW}$ of power on the Still and $125 \mu \mathrm{W}$ on the HEX/Cold Plate. The characterization tests demonstrated that the DU surpassed requested specifications. A MC cooling power of $\sim 10 \mu \mathrm{W}$ $(9.5 \mu \mathrm{W})$ at $12 \mathrm{mK}$ was obtained with $4 \mathrm{~mW}$ of power on the Still and $125 \mu \mathrm{W}$ on the HEX; nearly double the required cooling power.

Two Cerium Magnesium Nitrate (CMN) thermometers were calibrated during cool down using superconducting transition temperatures of a fixed point (FP) thermometer to ensure that the mixing chamber temperature was well understood. The lowest transition being tungsten at $\sim 14 \mathrm{mK}$.

\subsection{Complete CUORE Cryostat}

After successful independent commissioning the $4 \mathrm{~K}$ cryostat and the dilution unit were merged together and the complete cryogenic commissioning phase began. Following the installation of the inner stages (December 2013), the DU was removed from the test cryostat and inserted into the CUORE cryostat (January 2014). The first base temperature cold run began on March $7^{\text {th }}$ 2014, with the goal of reaching $10 \mathrm{mK}$

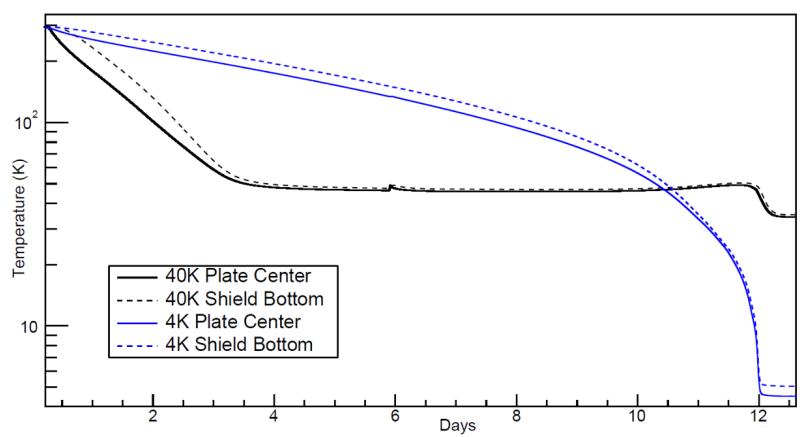

Figure 9: Temperature of the $40 \mathrm{~K}$ stage and $4 \mathrm{~K}$ as a function of time. Stable temperature of $35 \mathrm{~K}$ reached at the $40 \mathrm{~K}$ stage and $4.5 \mathrm{~K}$ at the $4 \mathrm{~K}$ stage with 4 pulse tubes. with no load. This was the first complete cryogenic cold run with all 6 thermal shields, the DU, support bars (down to the Still), thermalizations at each stage, thermometry and 4 out of 5 pulse tubes. This is a crucial step in commissioning to demonstrate the full operation of the cryostat at base temperature. Relative to the previous $4 \mathrm{~K}$-run the load at $4 \mathrm{~K}$ was doubled by the installation of the inner vessels and flanges, and one additional pulse tube installed. The cooldown time to $4 \mathrm{~K}$ was about 12 days, as seen in 
figure 9. The temperature of the $40 \mathrm{~K}$ stage was $35 \mathrm{~K}$ ( $2 \mathrm{~K}$ higher than before) and the temperature of the $4 \mathrm{~K}$ stage was $4.5 \mathrm{~K}(1.2 \mathrm{~K}$ higher than the previous run). The first base temperature run was very successful, reaching a stable temperature of $14 \mathrm{mK}$. In addition, the dilution unit performed extremely well, cooling the entire system from $4 \mathrm{~K}$ to base temperature in $\sim 12$ hours. When collecting the mixture a single-shot run was preformed reaching a temperature of $10.2 \mathrm{mK}$. During DU operation, the temperature of the Still stage should be $\sim 800 \mathrm{mK}$, but during the first base temperature run the Still temperature was $1.1 \mathrm{~K}$; higher than expected. This higher temperature corresponds to $\sim 25 \mathrm{~mW}$ of power being dissipated on the Still from thermal radiation. The cryostat was then warmed up and suspected problems addressed. A new cold run with improved baffles to solve the excess radiation problem is currently in preparation and will take place summer 2014.

\section{Conclusion}

CUORE will be the largest bolometric array ever attempted and the cryostat will be the largest ever operated at a temperature of $10 \mathrm{mK}$. Constructing and commissioning such a large cryostat is a challenge, a challenge intensified by the requirement to use only low-radioactivity materials. The CUORE cryostat is designed to overcome three main challenges: cooling down such a large mass to base temperature of $10 \mathrm{mK}$, reduction of vibrational noise transmitted to the detectors to ensure a good energy resolution, and background reduction through strict selection of cryostat materials. Both the $4 \mathrm{~K}$ cryostat and DU have independently met the required design specifications and the final commissioning phase of the complete CUORE cryostat has been underway since January 2014. At the time of writing the CUORE cryostat has successfully been commissioned to $14 \mathrm{mK}$ and a base temperature of $10 \mathrm{mK}$ or better is expected in the coming months.

\section{References}

[1] D. R. Artusa et al., Eur. Phys. J. C 74, 2956 (2014)

[2] F. Alessandria et al., Nucl.Instrum.Meth. A 727 (2013) 65-72

[3] A. Alessandrello et al., Nucl.Instrum.Meth. B 142 (1998) 163-172 